\section{Adaptive mixed image denoising based on image decomposition}

\author{
Run Liu, ${ }^{a}$ Shujun Fu, ${ }^{a}$ and Caiming Zhang ${ }^{b}$ \\ aShandong University, School of Mathematics, Jinan \\ 250100, China \\ bShandong University, School of Computer Science and \\ Technology, Jinan 250101, China \\ E-mail: shujunfu@163.com
}

\begin{abstract}
Image denoising while preserving image features is a key problem in image processing and computer vision. This letter proposes an adaptive mixed method for image restoration. First, this method decomposes a given image as the sum of two components: geometric structure and oscillating pattern according to Meyer's theory. Second, a coupled bidirectional diffusion equation is used to restore the structure part, and a nonlocal means filter is used to remove noise in the oscillating part. Experimental results show advantages of this method in feature-preserving denoising. (C) 2011 Society of Photo-Optical Instrumentation Engineers (SPIE). [DOI: 10.1117/1.3542041]
\end{abstract}

Subject terms: image denoising; image decomposition; anisotropic diffusion; shock filter; nonlocal filter; adaptive method

Paper 100761LR received Sep. 19, 2010; revised manuscript received Dec. 4, 2010; accepted for publication Dec. 27, 2010; published online Feb. 25, 2011.

\section{Introduction}

Image denoising while preserving image features such as edge, detail, and texture is a key problem in image processing and computer vision. In the past decade, variational approaches and partial differential equations have been widely used in image processing: ${ }^{1,2}$ for example, the total variation $(\mathrm{TV})^{3}$ and the anisotropic diffusion (AD) ${ }^{4}$ for image denoising, and the shock filter for image sharpening, ${ }^{5}$ etc. However, in the process of image denoising these algorithms based on variations and partial differential equations often blur fine details and particularly textures of image.

In 2005, Buades proposed a nonlocal means filter based on the information of a whole image, which can effectively preserve image details and textures. ${ }^{6}$ Due to relatively fewer pixels with similarity around edges, however, this method also blurs part of the image edges.

Observing the poor preservation of image texture by the TV denoising, Meyer proposed a TV- $G$ image decomposition model, ${ }^{7}$ where the $G$ space is used to model oscillating patterns instead of the Sobolev $L^{2}$ space. However, the $G$ norm is not easy to compute numerically. To overcome this difficulty, some algorithms are proposed to approximate Mayer's model. ${ }^{1,8}$

In this letter, to better preserve image details and textures, we propose an adaptive mixed method for image restoration. First, according to Meyer's theory, this method decomposes a given image into two components: the geometric structure and the oscillating pattern. Second, a coupled bidirectional diffusion equation 9 is used to restore the structure part, and a

0091-3286/2011/\$25.00 @ 2011 SPIE nonlocal means filter is used to remove noise in the oscillating part. The proposed method integrates advantages of image decomposition, the excellent capability of the shock filter in edge sharpening and that of the neighborhood filter in image texture denoising.

\section{Adaptive Mixed Image Restoration}

\subsection{Structure-Texture Image Decomposition}

Main information of an image is encoded in its edges, details, and textures. These components need to be represented in suitable spaces to capture their special characteristics in the image.

For a given image $f(x): \Omega \subset \mathbf{R}^{2} \rightarrow \mathbf{R}$, we decompose $f$ into the form $f=u+v$, where $u$ is the structure part including edges, details, and flat areas in $B V(\Omega),{ }^{1}$ and $v$ is the oscillating part including textures and noise in $G(\Omega) .^{7}$ In this letter, we adopt the following decomposition model: ${ }^{8}$

$$
\inf _{(u, v) \in B V(\Omega) \times G_{\mu}(\Omega)}\left[F(u, v)=\int_{\Omega}|D u|+\frac{1}{2 \lambda}\|f-u-v\|_{L^{2}}^{2}\right],
$$

where $G_{\mu}(\Omega)=\left\{v \in G(\Omega) \mid\|v\|_{G} \leq \mu\right\}$.

In a discrete framework Aujol et al. proved the existence and uniqueness of a solution to Eq. (1), and the approximation of Eq. (1) to Meyer's model. ${ }^{8}$

\subsection{Other Related Work}

In a report ${ }^{5}$ the special idea and technique developed in numerical solution of nonlinear hyperbolic equation were applied to feature-oriented image restoration for the first time. Then, Osher and Rudin introduced a novel edge sharpening technique called shock filter. ${ }^{5}$

In order to avoid unnatural artifacts of the shock-type processing, ${ }^{10}$ we introduced a soft edge sharpening algorithm in a coupled bidirectional diffusion (CBD) equation: ${ }^{9}$

$\frac{\partial u}{\partial t}=c_{N} u_{N N}+c_{T} u_{T T}-\omega_{N} \operatorname{sign}\left(G_{\sigma} * u_{N N}\right)|\nabla u|$,

where sign is a sign function, $\left(u_{N N}, u_{T T}\right)$ are the second directional derivatives along local normal and tangent directions, $G_{\sigma}$ is a Gaussian kernel function with the deviation $\sigma$, and $\left(c_{N}, c_{T}, \omega_{N}\right)$ are the forward and backward diffusion coefficients, respectively.

Another effective image denoising technique is the neighborhood filter, 6,11 which considers some similarity both in spatial location and in gray level of two pixels. Buades et al. proposed the following nonlocal means (NL-means) filter: ${ }^{6}$

$$
\begin{aligned}
& N L_{h} u(x) \\
& =\frac{1}{c(x)} \int_{\Omega} \exp \left[-\frac{\left\{G_{\sigma} *[u(x+\cdot)-u(y+\cdot)]^{2}\right\}(0)}{h^{2}}\right] \\
& \quad \times u(y) d y, \\
& \left\{G_{\sigma} *[u(x+\cdot)-u(y+\cdot)]^{2}\right\}(0) \\
& =\int_{\Omega} G_{\sigma}(z)[u(x+z)-u(y+z)]^{2} d z,
\end{aligned}
$$

where $c(x)=\int_{\Omega} \exp \left[-\left\{G_{\sigma} *[u(x+\cdot)-u(y+\cdot)]^{2}\right\}(0) /\right.$ $\left.h^{2}\right] d y$ is a normalization factor, and $h$ is a filtering parameter 


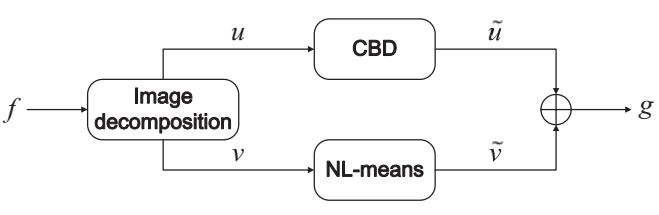

Fig. 1 The proposed mixed method.

related to noise level. The nonlocal means filter gives better results in denoising textured patterns.

\subsection{Proposed Mixed Model}

In order to better preserve image details and textures, we propose an adaptive mixed method based on image decomposition (AM-ID) for image restoration, which fuses different advantages of previously mentioned methods, such as the excellent capability of the shock filter in edge sharpening and that of the neighborhood filter in image texture denoising. This method decomposes a given image $f$ into two components: geometric structure $u$ such as edge and detail, and oscillating pattern $v$ including texture and noise according to Eq. (1). Then, the coupled bidirectional diffusion [Eq. (2)] is used to restore the structure part, and the nonlocal means filter [Eq. (3)] is used to remove noise in the oscillating part. For the sake of clarity, the AM-ID method is illustrated in Fig. 1.

Therefore, for a given degraded image $f$, we solve the following problem to reconstruct its restored version $g$ :

$\left\{\begin{array}{l}\inf _{(u, v) \in B V(\Omega) \times G_{\mu}(\Omega)}\left(F(u, v)=\int_{\Omega}|D u|+\frac{1}{2 \lambda}|| f-u-v \|_{L^{2}}^{2}\right) \\ \frac{\partial u}{\partial t}=c_{N} u_{N N}+c_{T} u_{T T}-\omega_{N} \operatorname{sign}\left(G_{\sigma} * u_{N N}\right)|\nabla u| \\ N L_{h} v(x)=\frac{1}{c(x)} \int_{\Omega} \exp \left[-\frac{\left\{G_{\sigma} *[v(x+\cdot)-v(y+\cdot)]^{2}\right\}(0)}{h^{2}}\right] v(y) d y \\ g=\tilde{u}+\tilde{v}\end{array}\right.$,

where $\tilde{u}$ and $\tilde{v}$ are solutions of the CBD and the NL-means processings, respectively.
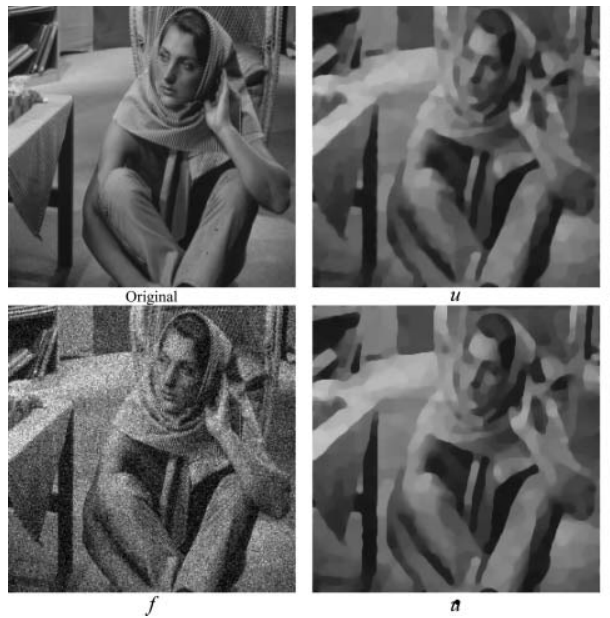

\section{Numerical Implementation and Experimental Results}

\subsection{Numerical Implementation}

In order to solve the model (4), we need to numerically compute Eqs. (1)-(3). For Eq. (1), we use the alternate projection algorithm. ${ }^{8}$ For Eq. (2), we use the numerically coupled scheme. ${ }^{9}$ Finally, for Eq. (3), a simple accelerating trick is adopted. ${ }^{12}$ On denoising oscillating patterns by the NLmeans method, a smaller parameter $h$ should be chosen to better preserve image textures.

\subsection{Experimental Results}

In this section we give some experimental results to show the performance of the proposed method. Although many test images are used, we only show experiments on two typical images (Barbara and Lena) because of the imposed page limit. The test images contain rich details, textures, and large scale features. The corrupted versions are obtained by adding a Gaussian noise.

In Fig. 2, we compare the proposed method (AM-ID) with the anisotropic diffusion (AD) on a noisy Barbara (256 $\times 256$ pixels) image. It is clear that, the given image has been successfully decomposd into the structure $u$ and oscillating patterns $v$ (texture and noise). At the same time, one can observe the edge sharpening of the CBD method in $\tilde{u}$, and the powerful denoising of the NL-means method in $\tilde{v}$. These processings have resulted in a better image restoration, where the noise is removed effectively, edges are sharpened reasonably, and most textures and details are preserved in $\tilde{u}+\tilde{v}$. As for the $\mathrm{AD}$ method, although it can effectively remove the noise, it has smoothed out most of image textures. Without distinguishing image texture from noise, it elminates both noise and textures.

In Fig. 3, we also test related methods on a noisy Lena $(256 \times 256$ pixels $)$ image. Once more, a better restoration of image textures and details is obtained by the proposed method. For example, see the feathers on the hat and Lena's eyes.

Finally, we report the peak signal to noise ratio $(\mathrm{dB})$ results and the computational time (seconds) in the parentheses, respectively: the noisy Barbara image (19.01), the AD method

Fig. 2 Denoising the noisy Barbara image (from top-left to bottom-right): Original $u, v+100$ by the method (1), result by the AD method, $f, \tilde{u}$ by the CBD processing, $\tilde{v}+100$ by the NL-means processing, result by the AM-ID method, respectively. 

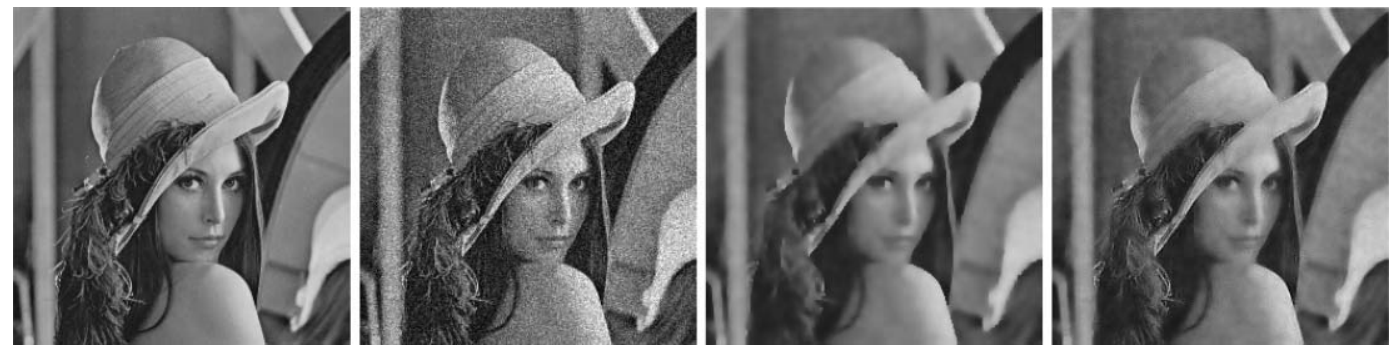

Fig. 3 Denoising the noisy Lena image (from left to right): original image, its noisy version, results by the AD and the AM-ID methods, respectively.

(20.42, 0.60), the AM-ID method $(22.08,6.56)$; the noisy Lena image (21.09), the AD method $(22.59,0.45)$, and the AM-ID method $(23.18,6.64)$. All methods are implemented using the MATLAB program on a notebook computer with $1.86 \mathrm{GHz}$ CPU and 3.00 GB Memory. Because of the complexity of the proposed method, a relatively longer computational time is needed.

\section{Conclusions}

Image denoising while preserving image features is an important task in image restoration, particularly for noisy textured images. This letter proposes an adaptive mixed method for image restoration, which fuses different advantages of several algorithms. Results in numerical experiments show that the proposed method produces better image restoration with effective noise removal and nice feature preserving of textures and details.

\section{Acknowledgments}

The research has been supported by the National Natural Science Foundation of China (Nos. 60933008, 61070094, 61020106001), and the China Postdoctoral Science Foundation (No. 20090460089).

\section{References}

1. G. Aubert and P. Kornprobst, Mathematical Problems in Image Processing: Partial Differential Equations and the Calculus of Variations, 2nd. ed. Springer-Verlag, New York (2006).

2. Y. Wang, R. Niu, X. Yu, L. Zhang, and H. Shen, "Image restoration and enhancement based on tunable forward-and-backward diffusion," Opt. Eng. 49, 057004 (2010).

3. L. Rudin, S. Osher, and E. Fatemi, "Nonlinear total variation based noise removal algorithms," Physica D 60, 259-268 (1992).

4. P. Perona and J. Malik, "Scale-space and edge detection using anisotropic diffusion," IEEE Trans. Pattern Anal. Mach Intell 12, 629639 (1990).

5. S. Osher and L. I. Rudin, "Feature-oriented image enhancement using shock filters," SIAM (Soc. Ind. Appl. Math.) J. Numer. Anal. 27, 919-940 (1990).

6. A. Buades, B. Coll, and J. M. Morel, "A review of image denoising algorithms, with a new one," Multiscale Model. Simul. 4, 490-530 (2005).

7. Y. Meyer, Oscillating Patterns in Image Processing and Nonlinear Evolution Equations, American Mathematical Society, Boston, (2001)

8. J. F. Aujol, G. Aubert, L. Blanc-Féraud, and A. Chambolle, "Image decomposition into a bounded variation component and an oscillating component," J. Math. Imaging Vision 22, 71-88 (2005).

9. S. Fu, Q. Ruan, C. Mu, and W. Wang, "Geometry-driven nonlinear equation with an accelerating coupled scheme for image enhancement," Lecture Notes Comput. Sci. 4487, 490-496 (2007).

10. S. Fu, Q. Ruan, W. Wang, F. Gao, and H. D. Cheng, "A featuredependent fuzzy bidirectional flow for adaptive image sharpening," Neurocomputing 70, 883-895 (2007).

11. D. Q. Chen, H. Zhang, and L. Z. Cheng, "Nonlocal variational model and filter algorithm to remove multiplicative noise," Opt. Eng. 49, 077002 (2010).

12. L. Condat, "A simple trick to speed up the non-local means," Tech Rep., GREYC Laboratory, Centre National de la Recherche Scientifique (CNRS), France (2010). 